\title{
Overnight studies in severe chronic left heart failure: arrhythmias and oxygen desaturation
}

\author{
S W Davies, L M John, J A Wedzicha, D P Lipkin
}

\begin{abstract}
Overnight studies were performed in $\mathbf{1 0}$ patients with severe chronic left heart failure (New York Heart Association grades III and IV) without pulmonary disease and in eight controls. Transcutaneous oxygen $\left(\mathrm{Po}_{2}\right)$ and carbon dioxide tensions $\left(\mathrm{PCO}_{2}\right)$ and oxygen saturation were measured and the electrocardiogram was recorded. During sleep mean oxygen saturation fell to $92 \cdot 7 \%$ (minimum $86.1 \%$ ) from $95 \cdot 1 \%$ when awake. During the night oxygen saturation was below $95 \%$ for $62 \%$ of the time, below $90 \%$ for $6 \%$ of the time, and below $85 \%$ for $1 \%$ of the time. In four patients there were oxygen desaturation dips (a fall of $>4 \%$ in oxygen saturation from a stable baseline that lasted $>30$ s) with concurrent increases in $\mathbf{P C O}_{2}$. Two patients had bradycardia during the dips: in one there was non-sustained ventricular tachycardia during the dips and in the other there was ST depression ( $>$ $0.1 \mathrm{mV}$ at $80 \mathrm{~ms}$ after the $J$ point) during a dip. In the controls the fall in mean oxygen saturation from $95.4 \%$ when they were awake to $94 \cdot 4 \%$ when they were asleep was less than the fall in patients with heart failure and there were no desaturation dips or arrhythmias.

Thus patients with severe heart failure had episodes of oxygen desaturation during sleep, some of which were associated with arrhythmia. Such episodes may be related to the increased risk of sudden death in chronic heart failure.
\end{abstract}

The prognosis in patients with severe chronic left heart failure (New York Heart Association grades III and IV) is poor, with a mortality of $40-50 \%$ at 12 months. ${ }^{1-3}$ Between one third and one half of these deaths are sudden, ${ }^{1-3}$ and this is true both of patients with end stage ischaemic heart disease and those with idiopathic dilated cardiomyopathy. ${ }^{14}$ Whatever the aetiology, many deaths in patients with severely impaired left ventricular function are sudden.

The pathophysiology of sudden death in severe chronic left heart failure is not known, but some or all of these deaths may be related to dangerous ventricular arrhythmias. ${ }^{5}$ Twenty four hour monitoring of the electrocardiogram showed frequent ventricular arrhythmias in such patients, ${ }^{6-8}$ and electrophysiological testing showed a low threshold for ventricular arrhythmias. ${ }^{9}$ Factors that

might trigger such arrhythmias during everyday life have not been fully defined, however.

Patients with severe chronic airflow obstruction had episodes of arterial oxygen desaturation during sleep ${ }^{10} 11$ that were associated with profound haemodynamic disturbance and arrhythmias. ${ }^{12}{ }^{13}$ Similar episodes might act as triggers for ventricular arrhythmias in chronic heart failure. We therefore performed overnight sleep studies in patients with chronic severe left heart failure to determine the presence of such episodes and their possible relation to arrhythmias.

\section{Patients and methods} PATIENT SELECTION

Overnight studies were performed on 10 patients (eight men and two women, aged 3376) with severe chronic left ventricular impairment and New York Heart Association grade III or grade IV dyspnoea. All were treated with diuretics (frusemide $80-160 \mathrm{mg} /$ day or its equivalent) and angiotensin converting enzyme inhibitors. All were clinically stable at the time of the study, without radiographic pulmonary oedema; four patients were on the waiting list for cardiac transplantation. The left ventricular ejection fraction determined by gated blood pool scanning was $<40 \%$ in all cases. Only one of the patients gave a history suggestive of sleep disordered breathing (snoring, daytime somnolence, and morning headache). None of the patients took sedatives at night.

We excluded patients with (a) evidence of important intrinsic pulmonary disease, as judged by history and clinical examination, normal peripheral lung fields on chest $x$ ray, and standard lung function tests (forced expiratory volume in one second, forced vital capacity, and carbon monoxide diffusing capacity) $>75 \%$ of predicted normal values; (b) current treatment with antiarrhythmic drugs; and (c) known ear, nose, or throat disease, or obesity ( $>115 \%$ ideal body weight). For comparison control data were obtained from eight healthy individuals (ages 32-75) referred to the sleep laboratory for investigation of possible sleep disordered breathing. All the controls had normal lung function and normal cardiac function (clinical examination, electrocardiogram, chest $x$ ray, lung function tests, echocardiogram). None of them had sleep disordered breathing on overnight study.

\section{OVERNIGHT STUDIES}

All those with chronic heart failure were inpatients undergoing assessment of heart
Bonner Road,

London E2 9JX.

Accepted for publication 11 October 1990 
failure at the time of the overnight study. Studies were performed at the bedside in the hospital ward on the third to eighth night after admission. Patients were thus familiar with the room and environment before the night on which the recordings were made. The following measurements were made: (a) transcutaneous oxygen tension $\left(\mathrm{Po}_{2}\right)$ by a Radiometer TCM2 electrode applied to anterior abdominal wall; $(b)$ transcutaneous carbon dioxide tension $\left(\mathrm{PCO}_{2}\right)$ by a Radiometer TCM20 electrode applied to anterior abdominal wall; $(c)$ oxygen saturation and heart rate from an Ohmeda Biox III pulse oximeter applied to a finger. The output from these devices was recorded on a four channel paper chart recorder (Honeywell Analogic).

Raw values of $\mathrm{PO}_{2}$ and $\mathrm{PCO}_{2}$ from the electrodes were corrected by a standard empirical formula validated for this laboratory. This standardisation was checked in each subject by comparing the values of transcutaneous $\mathrm{Po}_{2}$ and $\mathrm{PCO}_{2}$ with arterialised capillary blood samples from the ear lobe ${ }^{14}$ analysed in a Radiometer ABL3 machine at the start of the overnight study.

Heart rate, rhythm, and ST segment changes were recorded by a two channel Holter monitor with a wide band pass (Oxford Medilog mark II). The frequency response of $0.05-100 \mathrm{~Hz}$ meets American Heart Association specifications, ${ }^{15}$ and units were calibrated both before and after placement. Channel I recorded the lead with a configuration resembling that of precordial lead V5, and channel II recorded a lead with a configuration resembling that of standard lead III. Important ischaemic episodes were defined as transient ST segment depression $>0.1 \mathrm{mV}$ from the baseline at 80 ms after the $\mathrm{J}$ point, lasting for at least one minute. ${ }^{16}$ Each episode was confirmed by direct examination of the ST segments before, during, and after the episode on a full disclosure electrocardiogram. An interval of at least two minutes of normal tracing was required after resolution of each episode before another discrete episode was counted. ${ }^{16}$

We recorded synchronous time markers at the start and end of each study by the event marker channels of the Holter monitor and of the respiratory monitor to ensure accurate correlation between the two recordings. The accuracy of the time markers was confirmed by comparing heart rate changes on the electrocardiogram with those recorded on the paper chart recorder by the pulse oximeter.

\section{DATA ANALYSIS}

Overnight recordings of $\mathrm{Po}_{2}, \mathrm{PCO}_{2}$, and oxygen saturation were read by two experienced observers independently. Dips in oxygen saturation were defined as an abrupt fall in oxygen saturation by at least $4 \%$, lasting for at least 30 seconds. ${ }^{1718}$ We used standard criteria to identify episodes of ST segment depression, as given above. A significant episode of bradycardia was defined as a fall in mean heart rate by at least 10 beats per minute, so that the absolute heart rate fell below 60 beats per minute, lasting for at least 30 seconds.

\section{STATISTICAL ANALYSIS}

Values of variables are given as range and median; we used the appropriate nonparametric statistical tests in analyses. The group of patients with heart failure and the control group were compared by the MannWhitney $U$ statistic for continuous variables and Fisher's exact test for categorical variables. We used the Wilcoxon matched pairs signed rank test to compare measurements made when the patients were awake with those made when they were asleep.

\section{Results}

BLOOD GASES IN CHRONIC HEART FAILURE

At rest all patients with severe chronic left heart failure had normal lung volumes and gas transfer measurements and normal blood gases determined from arterialised capillary samples from the ear lobe (table). Mean oxygen saturation fell significantly from $95 \cdot 1 \%$ (range 93.2 $97 \cdot 0 \%)$ when they were awake to $92 \cdot 7 \%(89 \cdot 1-$ $94.8 \%)$ when they were asleep $(p<0.001)$. The minimum oxygen saturation during the overnight study in each patient was $86.1 \%$ (79.9-90.3\%). During the night arterial oxygen saturation was below $95 \%$ for $62 \%$ (range 40 $98 \%)$ of the time, below $90 \%$ for $6 \%(1-18 \%)$ of the time, and below $85 \%$ for $1 \%(0-5 \%)$ of the time. Mean carbon dioxide $\left(\mathrm{PCO}_{2}\right)$ rose from 34.1 (29.7-41.9) $\mathrm{mm} \mathrm{Hg}$ when patients were awake to $37.9(33.7-45.7) \mathrm{mm} \mathrm{Hg}$ when they were asleep $(p<0.005)$

In four patients there were significant dips with concurrent increases in $\mathrm{PCO}_{2}$. The number of overnight dips in each individual patient varied from two to eight (mean four). The duration of the dips ranged from one to four minutes. There were clusters of dips in two patients (fig 1).

Oscillations in oxygen saturation were recorded in two patients with chronic heart failure (fig 2). These had a frequency of between 0.45 and 0.8 cycles per minute, and episodes lasted between four and 17 minutes. There were synchronous but opposite changes in transcutaneous $\mathrm{PCO}_{2}$ consistent with cyclical changes in the depth of ventilation.

ELECTROCARDIOGRAM IN CHRONIC HEART FAILURE

The resting electrocardiogram was abnormal in all patients; all of them had anterior or inferior

Pulmonary function, body weight, and blood gases at rest (range (median)) in patients with severe chronic left heart failure and in controls

\begin{tabular}{lll}
\hline & $\begin{array}{l}\text { Chronic left } \\
\text { heart failure } \\
(n=10)\end{array}$ & $\begin{array}{l}\text { Controls } \\
(n=8)\end{array}$ \\
\hline Age & $33-76(58)$ & $32-75(56)$ \\
Weight $(\mathrm{kg})$ & $58-84(73)$ & $62-91(74)$ \\
$\mathrm{FEV}_{1}(1)$ & $1 \cdot 7-3 \cdot 2(2 \cdot 4)$ & $2 \cdot 0-4 \cdot 8(3 \cdot 3)$ \\
$(\mathrm{as} \%$ of predicted) & $76-113(84)$ & $80-125(98)$ \\
$\mathrm{FVC}(1)$ & $2 \cdot 1-4 \cdot 7(3 \cdot 1)$ & $3 \cdot 5-5 \cdot 5(4 \cdot 7)$ \\
$($ as \% of predicted) & $75-113(81)$ & $82-119(96)$ \\
$\mathrm{PaO}_{2}(\mathrm{~mm} \mathrm{Hg})$ & $68 \cdot 2-89 \cdot 6(79 \cdot 3)$ & $72 \cdot 9-89 \cdot 7(82 \cdot 6)$ \\
$\mathrm{PaCO}_{2}(\mathrm{~mm} \mathrm{Hg})$ & $29 \cdot 7-41 \cdot 9(34 \cdot 1)$ & $33 \cdot 1-40 \cdot 6(36 \cdot 0)$ \\
\hline
\end{tabular}

$\mathrm{FEV}_{1}$, forced expiratory volume in one second; FVC = forced vital capacity; $\mathrm{PaO}_{2}$, partial pressure of oxygen in arteria vital capacity; $\mathrm{PaO}_{2}$, partial pressure of oxygen in arterial
blood; $\mathrm{PaCO}_{2}$, partial pressure of carbon dioxide in arterial blood; 
Figure 1 An example of clustering of desaturation dips in a patient with

chronic heart failure.

Figure 2 Episode of oscillation in oxygen saturation and carbon dioxide in a patient with chronic heart failure.

Figure 3 Desaturation dip with associated episode of bradycardia in a patient with chronic heart failure.
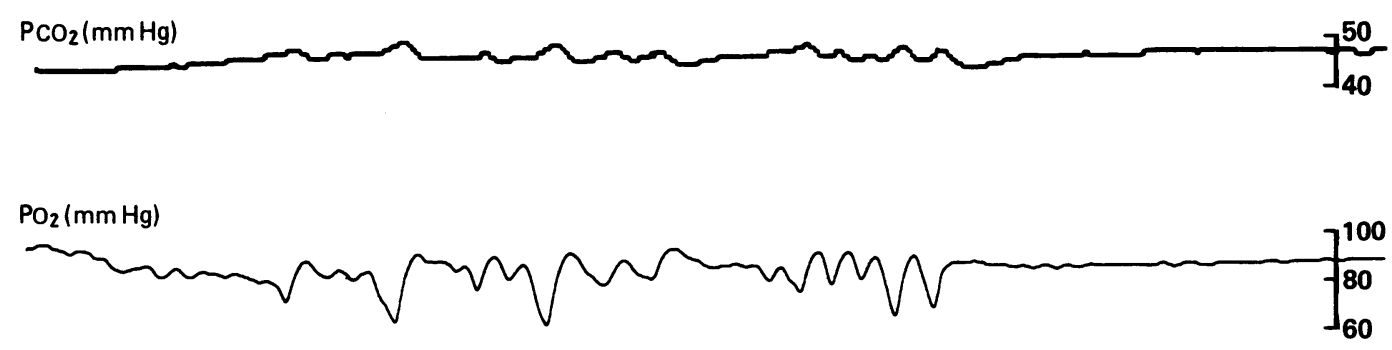

Oxygen saturation (\%)
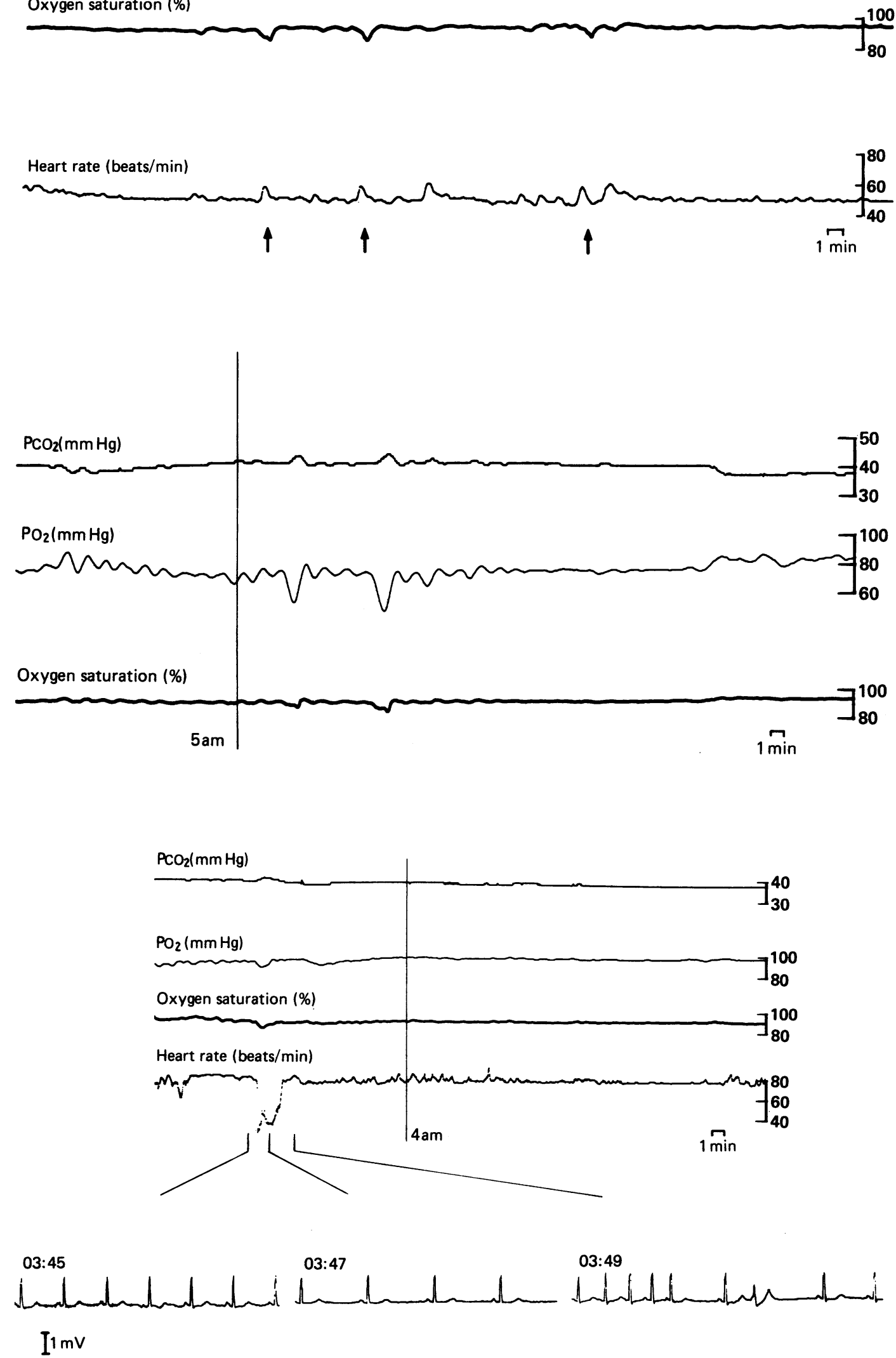
Figure 4 Desaturation dip with associated episodes of non-sustained ventricular tachycardia in a patient with chronic heart failure.

Figure 5 Desaturation dip with associated episode of transient $S T$ segment depression in a patient with chronic heart failure.
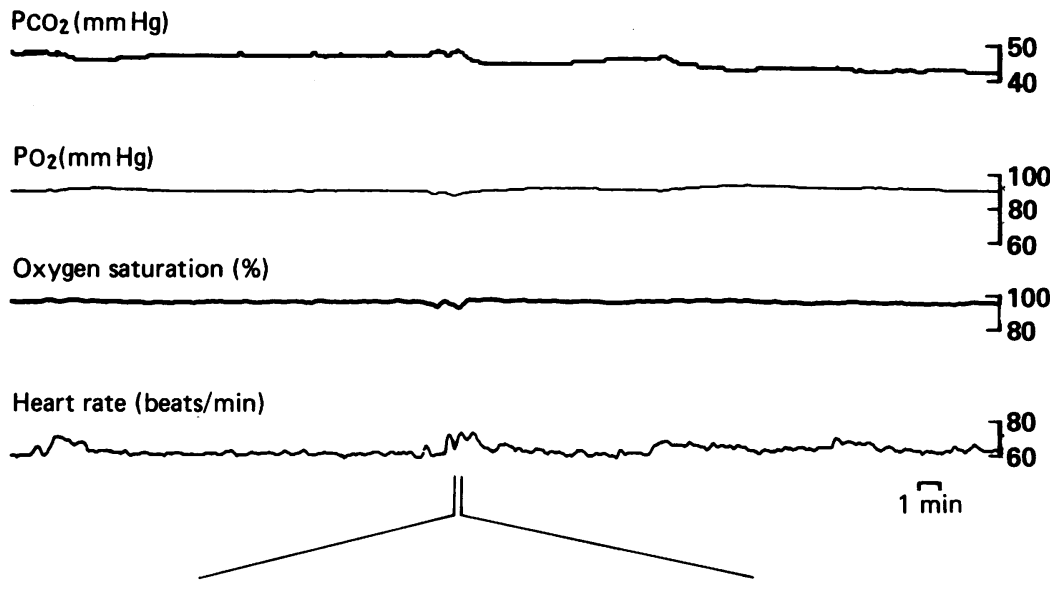

03:19

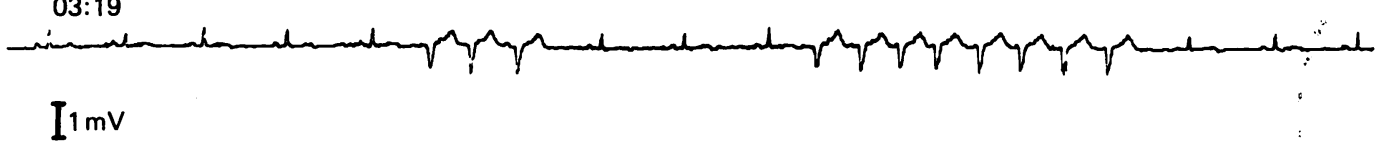

$Q$ wave infarction. ST segments were isoelectric on the resting 12 lead electrocardiogram. During sleep the mean heart rate decreased to 66 (10) bests per minute from 75 (8) beats per minute when patients were awake $(p<0.005)$.

In the six patients without arterial oxygen dips there were no arrhythmias apart from infrequent ventricular extrasystoles (more than three per minute). In two patients with desaturation dips there was transient bradycardia associated with the dips (fig 3 ). These were confirmed on both Holter monitoring and on the mean heart rate signal from the pulse oximeter. In one patient there were frequent ventricular extrasystoles and short runs of nonsustained ventricular tachycardia during dips (fig 4). No episodes of ventricular tachycardia were recorded in that patient at other times, nor in any other patient. In one patient there was

significant ST segment depression during one desaturation dip (fig 5).

The exact time relation of dip and arrhythmia is uncertain because the recordings were made on two separate systems. Synchronous time marks were placed on both recording systems at the start and at the end of each overnight study, and figures 3 and 4 show that the electrocardiographic changes in heart rate mirrored those recorded by the pulse oximeter during the dip. Heart rate during dips started to change within 30 seconds either side of the onset of the dip in oxygen saturation. Transient ST segment depression associated with one dip in a patient also coincided (within 30 seconds) with the onset of the dip.

CONTROL GROUP

Oxygen saturation while the eight age matched
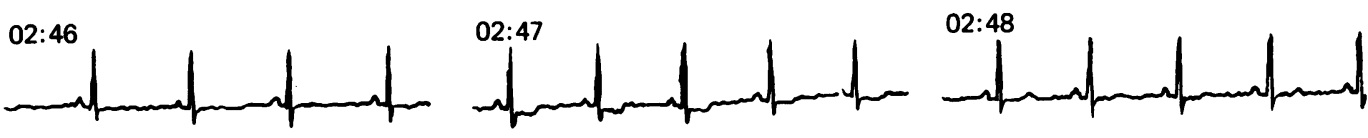

$I 1 \mathrm{mV}$
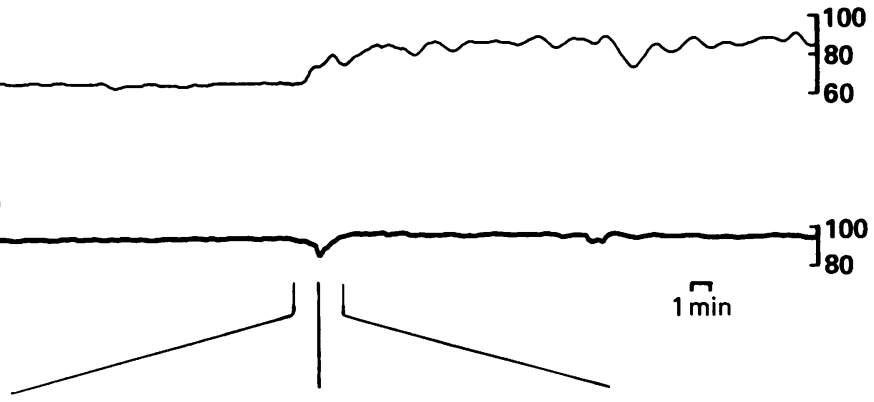

Oxygen saturation (\%)

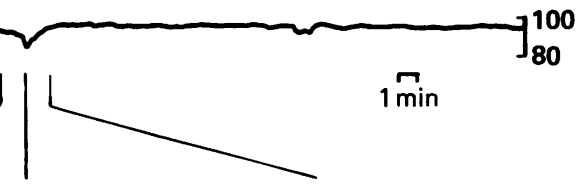


controls were awake was similar to that in the patients with heart failure. The fall in mean oxygen saturation overnight from $95.4 \%$ (range $93 \cdot 5-97 \cdot 2 \%$ ) to $94.4 \%(92 \cdot 4-96.5 \%)$, however, was less than in the patients with chronic heart failure $(p<0.01)$. There were no significant desaturation dips and no rhythm disturbances or shifts in ST segment.

\section{Discussion}

This study showed that the nocturnal decrease in arterial oxygen saturation and increase in $\mathrm{PCO}_{2}$ were greater in patients with severe chronic left heart failure than in controls. In some patients with heart failure there were abrupt dips in oxygen saturation, some of which were associated with arrhythmias or episodes of ST segment depression, or both.

The occurrence of sleep disordered breathing in severe chronic heart failure has not previously been defined. We did not deliberately select patients with chronic heart failure and symptoms of sleep disordered breathing and we excluded conditions that might contribute to sleep disordered breathing (pulmonary disease, obesity, nasopharyngeal conditions). Our results suggest that sleep disordered breathing may be common in patients with severe chronic heart failure.

\section{SLEEP DISORDERED BREATHING}

Changes in the respiratory pattern and in blood gas tensions during sleep were first recognised in patients with hypoxic lung disease..$^{1011}$ Episodes of arterial oxygen desaturation ("dips") in patients with chronic obstructive airways disease are associated with profound haemodynamic changes, ${ }^{1213}$ and may lead to sustained pulmonary hypertension, secondary polycythaemia, cor pulmonale, and impairment of the central nervous system. ${ }^{19} 20$ They may also indicate a poor prognosis. ${ }^{21}$ Sleep disordered breathing was also reported in patients with disorders of the upper airways, neuromuscular problems, obesity, and in some apparently healthy normal individuals. ${ }^{22}$

Two distinct mechanisms of apnoea account for the episodes of arterial oxygen desaturation. ${ }^{22}$ In central sleep apnoea, airflow at the nose and at the mouth stops for at least 10 seconds and there is a considerable reduction in respiratory movement. In obstructive sleep apnoea, airflow at the nose and mouth stops for at least 10 seconds and this is associated with increased respiratory effort and paradoxical movement of the chest wall. A mixture of both patterns may occur in an individual patient and there may also be episodes of hypopnoea that do not last for at least 10 seconds.

OVERNIGHT ARTERIAL OXYGEN DESATURATION IN CHRONIC LEFT HEART FAILURE

In the present study there was a small but significant fall in mean oxygen saturation during sleep in the patients with chronic heart failure and this was greater than was seen in the control group. The concomitant small rise in $\mathrm{PCO}_{2}$ suggests this was the result of a minor reduction in alveolar ventilation rather than shunting causing a venous admixture.

Significant desaturation dips with accompanying rises in $\mathrm{PCO}_{2}$ were seen in four of the 10 patients with severe chronic left heart failure and in none of the eight controls. There are several possible mechanisms for this in the patients with heart failure. Impaired cerebral perfusion and perhaps cerebral hypoxia might lead to central sleep apnoea. ${ }^{23}$ Decreased pharyngeal tone as a result of abnormalities of skeletal muscle ${ }^{2425}$ might cause intermittent obstruction of the upper airway. Further studies with full sleep staging are required to distinguish between these possibilities and to elucidate the mechanism of desaturation dips in chronic heart failure. The stage and depth of sleep may be important: in healthy adults ventilation and the ventilatory responses to hypoxia and hypocapnia are smaller during sleep than wakefulness, and are further reduced in rapid eye movement sleep. ${ }^{26}{ }^{27}$ Postural changes in the pulmonary ventilationperfusion relations in recumbent individuals may also contribute to nocturnal desaturation. $^{28}$

In two patients we found episodes of oscillation in oxygen saturation with synchronous opposite changes in $\mathrm{PCO}_{2}$. This was consistent with Cheyne-Stokes breathing, which has been described in severe heart failure with low output states. ${ }^{29}$ The oscillation may be the result of low cardiac output increasing the transit time from the pulmonary capillary bed to the carotid body and brain stem receptor and introducing a delay into the negative feedback system. ${ }^{30} 31$ Reductions in cardiac output during sleep might therefore be responsible for the cyclical oxygen saturation and $\mathrm{PCO}_{2}$ changes in the two patients in the present study.

\section{ARRHYTHMIAS}

In four of the 10 patients with severe chronic heart failure the overnight studies showed important arrhythmias including transient bradycardias, frequent ventricular extrasystoles and brief runs of ventricular tachycardia, and a transient left bundle branch block pattern. No rhythm disturbances were seen in any of the eight controls.

Both bradycardia and tachycardia were associated with oxygen desaturation dips, and often started within 30 seconds of the onset of a dip. Though the changes in oxygen saturation seen in this study were small, they could have acted synergistically with other factors to cause arrhythmias. In severe chronic left heart failure the threshold of the left ventricle to arrhythmia provoked by electrophysiological testing is low. ${ }^{9}$ Conversely, it is possible that changes in cardiac output caused by the rhythm disturbance or myocardial ischaemia reduced cerebral perfusion and led to a change in respiratory pattern and to transient hypoventilation. Finally, a transient disturbance of brain stem function might affect autonomic outflow and hence rhythm while simultaneously affecting the respiratory centres and hence the ventilatory drive. There is insufficient evidence to decide between these 
possibilities, and we cannot at present assign a causal role to the oxygen saturation dips in triggering the arrhythmias we saw.

\section{CLINICAL IMPLICATIONS}

Sleep disordered breathing is thought to affect the quality of life of patients with chronic airflow obstruction by disrupting the normal temporal structure of sleep and by contributing to specific symptoms such as nocturnal dyspnoea, general fatigue, malaise, and irritability. The same may apply to patients with severe heart failure. We can also speculate that the cumulative effects of repeated small oxygen saturation dips might in the long term influence myocardial and cerebral function. Though the dips and the overall nocturnal decrease in mean oxygen saturation in heart failure are smaller than in patients with respiratory disorders ${ }^{1011}$ the failing heart may be more sensitive to oxygen desaturation.

Because the occurrence of arrhythmias during everyday life may predict a poor prognosis in chronic heart failure, ${ }^{32}$ the association between oxygen saturation dips and arrhythmias (and in one case significant ST segment depression) is of interest. Nobody knows whether the dips in oxygen saturation are causally related to arrhythmias and ischaemia or whether they both reflect brainstem hypoperfusion and autonomic instability. Further study of these dips may provide insight into the mechanisms of sudden death in chronic heart failure. Though the definition of sudden cardiac death in surveys is arbitrary, ${ }^{33}{ }^{34}$ it does include deaths without any antecedent deterioration of cardiac function which are thought to be the result of arrhythmia. The factors that trigger arrhythmias in severe chronic heart failure have not been fully defined, but small changes in oxygen saturation might contribute.

There are circadian rhythms in the time of onset of myocardial infarction ${ }^{35}$ and sudden cardiac death. ${ }^{36}$ No such pattern has yet been described for sudden death in severe heart failure, but the present findings suggest a mechanism for an excess of nocturnal deaths. This would differ in mechanism and in timing from the early morning peak in myocardial infarction that parallels the normal physiological rhythms of blood pressure, platelet aggregability, fibrinogen concentrations, and plasma catecholamines. ${ }^{35} 36$

Various treatments have been tried for the different forms of sleep disordered breathing. Reducing alcohol intake, withholding hypnotic and sedative drugs, and weight reduction for the obese patient have proved beneficial in selected cases. There is a single case report of sleep disordered breathing in a patient with severe chronic heart failure that resolved when the chronic heart failure was controlled by intensive diuretic treatment. ${ }^{37}$ It is too early to advocate more specific treatment for sleep disordered breathing in patients with chronic heart failure. Two recent studies report the apparent benefits of supplemental oxygen ${ }^{38}$ and of nasal continuous positive airway pressure ${ }^{39}$ in patients with chronic heart failure and overt Cheyne-Stokes respiration.

\section{CONCLUSION}

In a group of 10 patients with severe chronic left ventricular failure (chronic heart failure) the decrease in mean arterial oxygen saturation and increase in $\mathrm{PCO}_{2}$ were both slightly greater than in a control group of eight subjects. In four of the 10 patients with chronic heart failure there were abrupt dips in oxygen saturation during sleep, some of which coincided with arrhythmias or transient ST segment depression or both. The significance of these abnormalities has yet to be evaluated. They may contribute to various symptoms and to the high risk of sudden death in patients with chronic heart failure. Patients with severe heart failure are potentially at risk of arterial oxygen desaturation and may require overnight oximetry to define the degree of respiratory, sleep, and rhythm disturbance.

During this study SWD was supported by a British Hear Foundation junior research fellowship.

1 Franciosa JA, Wilen $M$, Ziesche $S$, Cohn JN Survival in men with severe chronic left ventricular failure due to either coronary heart disease or idiopathic dilated cardiomyopathy. Am J Cardiol 1983;51:831-6.

2 Cohn JN, Levine TB, Olivari MT, et al. Plasma norepinephrine as a guide to prognosis in patients with chronic congestive heart failure. N Engl J Med 1984;311:819-23.

3 Packer M. Sudden unexpected death in patients with congestive heart failure: a second frontier. Circulation 1985;72:681-5

4 Francis GS. Development of arrhythmias in the patients with congestive heart failure: pathophysiology, prevalence and prognosis. Am J Cardiol 1986;57:3B-7B.

5 Cohn JN, Archibald DG, Ziesche S, et al. Effect of vasodilator therapy on mortality in chronic congestive heart failure: results of a Veterans Administration Cooperative Study (V-HeFT). $N$ Engl J Med 1986; 314:1547-52.

6 Von Olshausen K, Schäfer A, Mehmel HC, Schwarz F, Senges J, Kübler W. Ventricular arrhythmias in idiopathic dilated cardiomyopathy. Br Heart J 1984; 51:195-201.

7 Meinertz T, Hoffman T, Kasper W, et al. Significance of ventricular arrhythmias in idiopathic dilated car diomyopathy. Am J Cardiol 1984;53:902-7.

8 Maskin CS, Siskind SJ, LeJemtel TH. High prevalence of non-sustained ventricular tachycardia in severe congestive heart failure. Am Heart $J$ 1984;107:896-901.

9 Spielman SR, Greenspan AM, Kay HR, et al. Electrophysiological testing in patients at high risk of sudden death. I. Nonsustained ventricular tachycardia and abnormal ventricular function. J Am Coll Cardiol 1985;6:31-9.

10 Flick MR Block AJ. Continuous in vivo monitoring of arterial oxygenation in chronic obstructive lung disease. Ann Intern Med 1977;86:725-30.

11 Douglas NJ, Calverley PMA, Leggett RJE, Brash HM, Fenley DC, Brezinova V. Transient hypoxaemia during Fenley DC, Brezinova V. Transient hypoxaemia during sleep in $1979 ; \mathbf{i}: 1-4$.

12 Coccagna G, Lugaresi E. Arterial blood gases and pulmonary and systemic pressure during sleep in chronic obstructive pulmonary disease. Sleep 1978;1:117-24.

13 Boysen PG, Block AJ, Wynne JW, Hunt LA, Flick MR. Nocturnal pulmonary hypertension in patients with chronic obstructive pulmonary disease. Chest 1979;76:536-42.

14 Spiro SG, Dowdswell IRG. Arterialised ear lobe blood samples for blood gas tensions. Br J Dis Chest 1976; 70:263-8.

15 Pipberger HV, Arzbaecher RC, Berson AS, et al. American Heart Association Committee Report: recommendations for standardisation of leads and specifications for insfor standardisation of leads and specifcations for insCirculation 1975;52(suppl II):II.11-31.

16 Gottlieb SO, Weisfeldt ML, Ouyang $\mathrm{P}$, et al. Silent ischaemia as a marker for early unfavourable outcomes in ischaemia as a marker for early unfavourable outcomes in patients wit w-9.

17 Block AJ, Boysen PG, Wynne JW, Hunt LA. Sleep apnoea, hypopnoea and oxygen desaturation in normal subjects. A hypopnoea and oxygen desaturation in normal subjects. A
strong male predominance. N Engl J Med 1979;300:513-7. strong male predominance. $N$ Engl J Med 1979;300:513-7.
American Thoracic Society. Indications and standards for cardiopulmonary sleep studies. Am Rev Respir Dis cardiopulmonary

19 Block AJ, Boyse PG, Wynne JW. The origins of cor 
pulmonale: a hypothesis. Chest 1979;75:109-10.

20 Flenley DC. Clinical hypoxia: causes, consequences, and correction. Lancet 1978;i:542-6.

21 He J, Kryger MH, Zorick FJ, Conway W, Roth T. Mortality and apnea index in obstructive sleep apnea. Chest 1988;94:9-14.

22 Strohl KP, Sullivan CE, Saunders NA. Sleep apnea syndromes. In: Saunders NA, Sullivan CE, eds. Sleep and breathing (lung biology in health and disease, volume 19). New York: Marcel Dekker, 1984:365-402.

23 Plum F, Leigh RJ. Abormalities of central mechanisms. In: Hornbein TF, ed. Regulation of breathing, part II (lung biology in health and disease, volume 17.) New York: biology in health and disease,

24 Remmers JE, de Groot WJ, Sauerland EK, Anch AM. Pathogenesis of upper airway occlusion during sleep. $J$ Appl Physiol 1979;44:931-8.

25 Hudgel DW, Martin RJ, Hill P. Mechanics of the respiratory system and breathing pattern during sleep in normal humans. J Appl Physiol 1986;56:133-7.

26 Berthon-Jones M, Sullivan CE. Ventilatory and arousal responses to hypoxia in sleeping humans. Am Rev Respir Dis 1982;125:632-9.

27 Douglas MJ, White DP, Weil JV, Pickett CK, Zwillich CW. Hypercapnic ventilatory response in sleeping adults. $\mathrm{Am}$ Rev Respir Dis 1982;126:530-3.

28 Kaneko K, Milic-Emili J, Dolovich MB, Dawson A, Bates DV. Regional distribution of ventilation and perfusion as a DV. Regional distribution of ventilation and perfusion as a
function of body position. J Appl Physiol 1966;21:767-77.

29 Pryor WW. Cheyne-Stokes respiration in patients with ryor WW. Cheyne-Stokes respiration in patients with
cardiac enlargement and prolonged circulatory time. Circulation 1951:4.233-8.

30 Lange RL, Hecht $\mathrm{HH}$. The mechanism of Cheyne-Stokes respiration. J Clin Invest 1962;41:42-52.

31 Khoo MCK, Kronauer RE, Strohl KP, Slutsky AS. Factors inducing periodic breathing in humans: a general model. $J$ Appl Physiol 1982;53:644-59.

32 Holmes J, Kubo SH, Cody RJ, Kligfield P. Arrhythmias in ischemic and non-ischemic dilated cardiomyopathy: prediction of mortality by ambulatory electrocardiography. Am J Cardiol 1985;55:146-51.

33 Corday E. Symposium on identification and management of the candidate for sudden cardiac death. Introduction. $\mathrm{Am}$ the cardiol 1977;39:813-5.

34 Greene HL, Richardson DW, Barker AH, and the CAPS Investigators. Classification of deaths after acute myocarInvestigators. Classification of deaths after acute myocarCardiac Arrhythmia Pilot Study). Am J Cardiol 1989; 63:1-6.

35 Muller JE, Stone PH, Turi ZG, et al. Circadian variation in the frequency of onset of acute myocardial infarction. $N$ Engl J Med 1985;313:1315-7.

36 Muller J, Ludmer PL, Willich SN, et al. Circadian variation in the frequency of sudden cardiac death. Circulation 1987;75:131-5.

37 Baylor P, Tayloe D, Owen D, Sanders C. Cardiac failure presenting as sleep apnoea. Elimination of apnoea following medical management of cardiac failure. Chest 1988;94:1298-300.

38 Hanley PJ, Millar TW, Steljes DG, et al. The effect of oxygen on respiration and sleep in patients with congestive oxygen on respiration and sleep in patients with cong

39 Takasaki Y, Orr D, Popkin J, Rutherford R, Liu P, Bradley TD. Effect of nasal continuous positive airway pressure on sleep apnoea in congestive heart failure. Am Rev Respir Dis sleep apnoea in congest 